\title{
A pilot study of a novel variable-stiffness stylet for efficient colonoscope insertion with ex-vivo colon model
}

\author{
Daisuke Kikuchi, Ryusuke Kimura, Kosuke Nomura, Masami Tanaka, Yorinari Ochiai, Toshiro Iizuka and Shu Hoteya \\ Department of Gastroenterology, Toranomon Hospital, Tokyo, Japan
}

\begin{abstract}
:
Objectives: The variable-stiffness colonoscope is reportedly useful for making colonoscope insertion easier. However, this function is not associated with all colonoscopes. We developed a variable-stiffness stylet that can be inserted into the endoscope instrumentation channel to change the rigidity of the endoscope. Methods: We developed a stylet with adjustable stiffness and investigated its utility in colonoscope insertion using an ex-vivo model. Four endoscopists performed 24 colonoscope insertions, alternating between using the stylet (Stylet method) and the conventional method. We assessed insertion rate, rate of applying abdominal compression, and insertion time between the two groups. Results: In all procedures, the endoscope was inserted up to the cecum. There were significantly fewer external abdominal compressions with the Stylet method $(1 / 12,8.3 \%)$ compared to the conventional method $(6 / 12,50 \%)$. The insertion time was shorter with the Stylet method $(140.9 \pm 53.7 \mathrm{~s})$ compared to the conventional method $(181.3 \pm 64.9 \mathrm{~s})$. Conclusions: Using the variable-stiffness stylet, currently under development, resulted in significantly fewer external abdominal compressions and tended to have shorter insertion time.
\end{abstract}

Keywords:

colonoscopy, insertion, variable stiffness

J Anus Rectum Colon 2019; 3(3): 116-120

\section{Introduction}

Since colonoscopy is more widely available, colon cancer is now being detected at earlier stages, and patients undergo timely endoscopic treatment. Early and accurate detection of colon cancer is important for reducing mortality. Appropriate colonoscope insertion is essential for colon cancer detection, yet requires skill and good procedural technique.

In terms of specific technique, when advancing the colonoscope to intubate the cecum, it is important to avoid overstretching the intestine. Ideally, a technique that prevents loop formation should be used. However, in patients with a history of abdominal surgery or adhesions, or in female or elderly patients, looping may occur. The variable-stiffness colonoscope was developed because scope flexibility is required when loop formation occurs, and rigidity is desired for insertion into the proximal colon so that the scope does not re-loop deep inside the colon. There are several reports of the variable-stiffness colonoscope's efficacy ${ }^{1,2)}$, yet it is not a function associated with all colonoscopes.

We developed a variable-stiffness stylet, which can be inserted into the endoscope instrumentation channel to increase the rigidity of the endoscope, and investigated its utility.

\section{Methods}

\section{Variable-stiffness stylet}

We developed a stylet with adjustable stiffness in collaboration with Tokusen Kogyo Co (Hyogo, Japan). It measured $2.5 \mathrm{~mm}$ in diameter, length $1 \mathrm{~cm}$ (each cylinder), and it was 


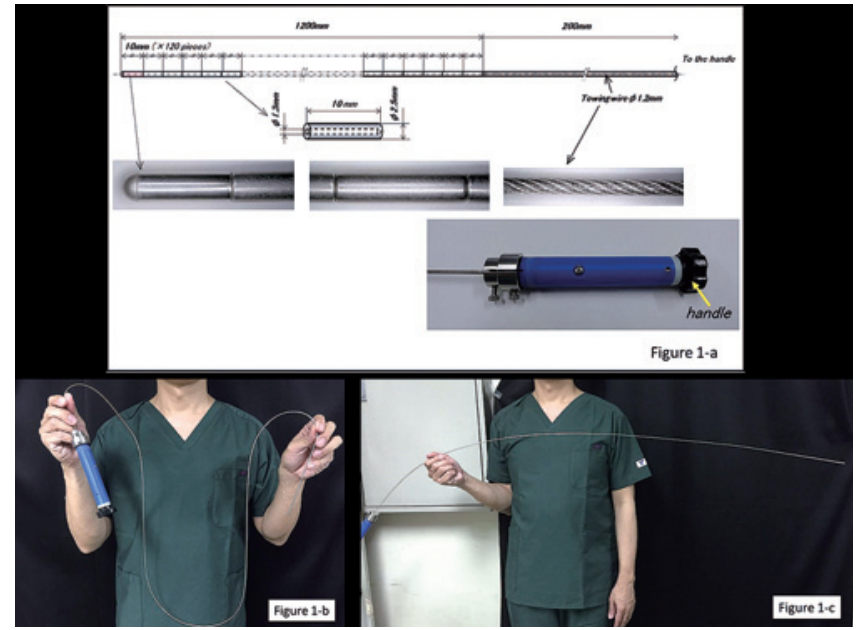

Figure 1. Photograph of the variable-stiffness stylet.

(a) Schematic diagram. Turning the handle, indicated by the yellow arrow, leads to interlocking the 1-cm stainless-steel cylinders, resulting in a rigid tube.

(b) Stylet in flexible state.

(c) Stylet in rigid state. composed of 120 interconnected stainless-steel cylindrical structures, with a wire affixed to the first cylinder. The stylet's total length was $155 \mathrm{~cm}$. The wire was passed through the insides of the cylinders such that, when the handle attached to the wire is turned, the cylindrical structures interlock to become a rigid catheter to increase the colonoscope's stiffness. Turning the handle in the opposite direction causes the lock to be released, and the stylet becomes flexible (Figure 1).

Regarding the method of use, the stylet is first inserted into the instrumentation channel. If the handle is not turned, the endoscope's flexibility does not change from its initial state. When the handle is turned, the endoscope stiffens, and when the handle is turned the other way, the endoscope returns to the flexible state (Figure 2).

\section{Ex-vivo model (Figure 3)}

We performed experiments in an ex-vivo model to examine the utility of the variable-stiffness stylet in colonoscope insertion. Four endoscopists performed colonoscope inser-
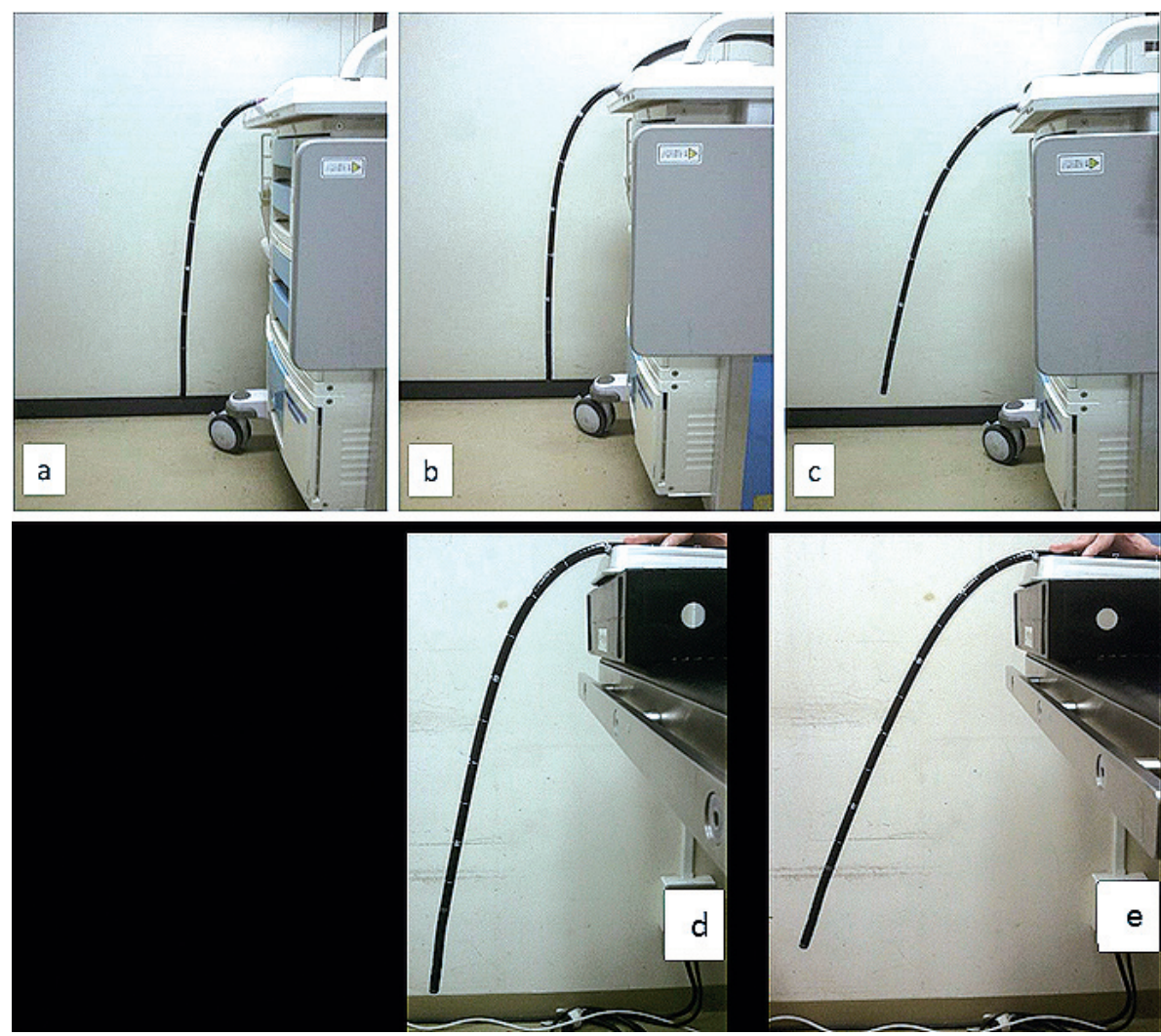

Figure 2. Comparison of the colonoscope to stylet and variable-stiffness colonoscope currently commercially available.
(a) Without stylet.
(b) With stylet in flexible state.
(c) With stylet in rigid state.
(d) Variable-stiffness colonoscope at maximum flexibility.
(e) Variable-stiffness colonoscope at maximum regidity. 
tion. All were board-certified endoscopists of the Japan Gastroenterological Endoscopy Society. Alternating between using the stylet (Stylet method) and the conventional method, they performed colonoscope insertion 24 times. Two endoscopists performed the conventional method first, then the Stylet method. The other two endoscopists performed the Stylet method first, followed by the conventional method. The CF Q240 (Olympus, Tokyo, Japan) endoscope was used in the experimental Colonoscopy Training Model (Kyoto Kagaku Co. Ltd., Kyoto, Japan). The ex-vivo colon model's technical difficulty ranged from level 1 (the easiest) to 5 (the most difficult), and it was set to level 5 in this study. If the intestines were overstretched and insertion into the proximal colon became difficult, external abdominal compression was permitted as necessary. In detail, when insertion remained difficult due to overstretching of the same part of the intestine after three consecutive attempts, external abdominal compression was applied. In the Stylet method, af-

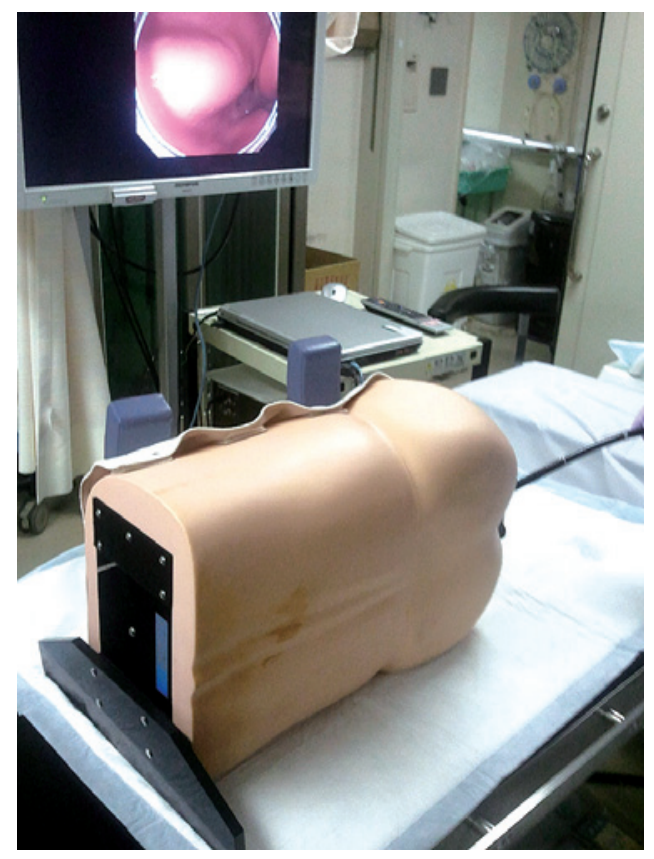

Figure 3. Colonoscopy training model used in this study. ter inserting the stylet, the endoscope was initially inserted in the flexible state, then the stiffness was changed to rigid as deemed necessary.

The time required to aspirate $100 \mathrm{ml}$ of water was evaluated in each methods. We conducted this suction trial three times each.

\section{Evaluation items}

In this study, we evaluated the insertion rate, insertion time, rate of applying external abdominal compression, insertion time from the anus to the splenic flexure (SF), and insertion time from the SF to the cecum. We also evaluated the suction time of $100 \mathrm{ml}$ of water. Insertion time was defined as the time taken for the endoscope to pass from the anus to the cecum.

\section{Statistical analysis}

Data were analyzed using the unpaired $t$-test, Chi-squared test, Fisher's test, or Mann-Whitney U-test as appropriate. A $P$ value less than 0.05 was considered significant. All statistical analyses were performed using SPSS version 20 (SPSS IBM statistics).

\section{Results}

The study results were shown in Table 1 . In all 24 trials, the endoscope was inserted up to the cecum. The insertion time was $181.3 \pm 64.9 \mathrm{~s}$ with the conventional method and was shorter with the Stylet method, at $140.9 \pm 53.7 \mathrm{~s}(\mathrm{p}=$ 0.17). Significantly fewer abdominal compressions were applied when using the Stylet method compared to the conventional method [1/12 (8.3\%) vs. 6/12 (50\%), respectively; $\mathrm{P}=$ 0.03]. Insertion time up to the $\mathrm{SF}$ in the conventional and Stylet methods were essentially the same, at $52.9 \pm 27.2 \mathrm{~s}$ and $53.3 \pm 23.9$ s, respectively. In contrast, insertion time from the SF up to the cecum was $128.4 \pm 56.9 \mathrm{~s}$ with the conventional method, and was shorter with the Stylet method, at $87.7 \pm 64.0 \mathrm{~s}(\mathrm{p}=0.11)$.

The suction time with the Stylet method was $22.2 \pm 2.3 \mathrm{~s}$, which was significantly longer compared to the conventional

Table 1. The Results of This Study.

\begin{tabular}{lcrl}
\hline & Conventional method & Stylet method & \\
\hline Insertion rate, $\%(\mathrm{n})$ & $100(12 / 12)$ & $100(12 / 12)$ & \\
\hline Insertion time, $\mathrm{s}( \pm \mathrm{SD})$ & $181.3 \pm 64.9$ & $140.9 \pm 53.7$ & $\mathrm{P}=0.17$ \\
$\quad$ From anus to SF & $52.9 \pm 27.2$ & $53.3 \pm 23.9$ & $\mathrm{P}=0.93$ \\
$\quad$ From SF to cecum & $128.4 \pm 56.9$ & $87.7 \pm 64.0$ & $\mathrm{P}=0.11$ \\
\hline External abdominal compression, \% $(\mathrm{n})$ & $50(6 / 12)$ & $8.3(1 / 12)$ & $\mathrm{P}=0.03$ \\
\hline Suction time, $\mathrm{s}( \pm \mathrm{SD})$ & $7.9 \pm 0.9$ & $22.2 \pm 2.3$ & $\mathrm{P}=0.02$ \\
\hline SD: Standard deviation & & & \\
SF: Splenic flexure & & &
\end{tabular}




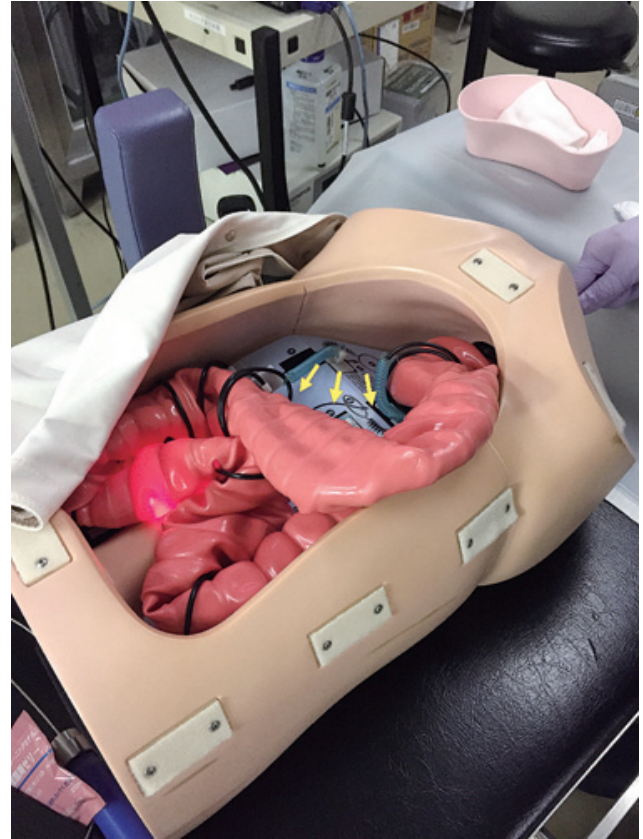

Figure 4. Picture of the training model during insertion at the transverse colon. Loop formation of sigmoid colon is observed. The direction of overstretching of the sigmoid colon was indicated with a yellow allow.

method $(7.9 \pm 0.9 \mathrm{~s}, \mathrm{p}=0.02)$.

\section{Discussion}

Colonoscopy can cause pain, often due to excessive intestinal stretching. Many studies have examined the insertion and pain associated with colonoscopy ${ }^{3)}$. Age, body mass index, history of abdominal procedures, and sex are reported to be related to coloscopy-associated pain. Jia et al. ${ }^{4)}$ used a scoring system to categorize colonoscope insertion difficulty and reported that pain can be predicted. The present study was at a preclinical stage, using a colonoscopy training model; thus, we could not evaluate pain. As soon as the variable-stiffness stylet is approved for clinical use, we hope to examine the relationship between stylet use and pain.

Various efforts are being made to alleviate the pain associated with colonoscopy. One is appropriate sedation ${ }^{5,6}$. Propofol, in particular, is often used in endoscopy in recent years. However, sedatives have disadvantages, such as the potential for adverse events and the need for monitoring and subsequent recovery. Second, $\mathrm{CO}_{2}$ delivery may be effective in reducing abdominal discomfort and pain ${ }^{7}$. Also, using a transparent hood on the tip of the endoscope or the water exchange method are considered effective ${ }^{8,9)}$. In the present study, we did not use a hood, and we used delivery of normal air. In clinical practice, we plan to investigate the stylet's effect on pain reduction in combination with other methods.
Various modifications and improvements in endoscopes and related equipment, such as variable-stiffness colonoscopies $^{1,22}$, ultrathin colonoscopies ${ }^{10-12)}$, passive bending endoscopes $^{13)}$, and double balloon enteroscopes ${ }^{14)}$, have been developed to facilitate colonoscope insertion. Specifically, endoscopes with adjustable stiffness capabilities are widely used. Typically, the endoscope becomes flexible when a loop is forming in the sigmoid colon. However, when inserting further than the SF, it is important not to form another loop at the sigmoid. Thus, a rigid scope is used when advancing further (Figure 4). A meta-analysis revealed that endoscopes with variable-stiffness capabilities improved insertion rates and reduced need for procedures, such as abdominal compression and position change ${ }^{1,2}$. There was reportedly no significant difference in terms of insertion time between endoscopes with and without variable stiffness; however, a subgroup analysis of adults reported that variablestiffness colonoscopes had a significantly shorter insertion times. A colonoscope with this stylet has almost same appearance as variable-stiffness colonoscopes that are currently commercially available (Figure 2). In the present study, using the stylet reduced insertion time and application of external abdominal pressure, suggesting that the stylet may have similar effects as the variable-stiffness colonoscopes currently used in clinical practice. Furthermore, using this stylet in clinical practice will enable all endoscopes to have variable-stiffness capabilities. The stylet could potentially be used in endoscopes other than colonoscopies, such as double balloon enteroscopes and ultrathin endoscopes, and even in non-endoscopic procedures such as an ileus tube.

The present study has several limitations. First, this study used an ex-vivo model with a small sample size. We thought using this highly realistic model was suitable for a preclinical study because its usefulness in endoscopy training has already been reported ${ }^{15}$. However, equipment that can be used effectively in clinical practice must be developed, and its efficiency must be investigated in a large-scale prospective trial. Moreover, because the stylet is inserted into the instrumentation channel, suction force might decrease. In this study, the suction time in the Stylet method was more than twice as long as the conventional method. Aspiration of solid intestinal contents may be more difficult and considered to be a big problem in clinical practice. The stylet diameter is $2.5 \mathrm{~mm}$, so reducing the caliber further may result in diminished efficiency. We plan to investigate the optimal caliber necessary for developing the stylet.

In conclusion, using the variable-stiffness stylet, currently under development, resulted in significantly fewer external abdominal compressions and tended to have shorter insertion times. We intend to continue developing this stylet for use in clinical practice. 


\section{Acknowledgments}

The prototype was designed and produced by Tokusen Kogyo Co.

\section{Conflicts of Interest}

There are no conflicts of interest.

\section{References}

1. Xie Q, Chen B, Liu L, et al. Does the variable-stiffness colonoscope makes colonoscopy easier? A meta-analysis of the efficacy of the variable stiffness colonoscope compared with the standard adult colonoscope. BMC Gastroenterol 2012; 12: 151.

2. Othman MO, Bradley AG, Choudhary A, et al. Variable stiffness colonoscope versus regular adult colonoscope: meta-analysis of randomized controlled trials. Endoscopy 2009; 41: 17-24.

3. Bugajski M, Wieszczy P, Hoff G, et al. Modifiable factors associated with patient-reported pain during and after screening colonoscopy. Gut 2017 (in press).

4. Jia H, Wang L, Luo H, et al. Difficult colonoscopy score identifies the difficult patients undergoing unsedated colonoscopy. BMC Gastroenterol 2015; 15: 46.

5. Padmanabhan A, Frangopoulos C, Shaffer LET. Patient Satisfaction With Propofol for Outpatient Colonoscopy: A Prospective, Randomized, Double-Blind Study. Dis Colon Rectum 2017; 60: 1102-1108.

6. Lovett P, Gómez V, Hodge DO, et al. Propofol Versus Midazolam/ Fentanyl Sedation for Colonoscopy in the Elderly Patient Population. J Perianesth Nurs 2017; 32: 210-214.

7. Cadoni $S$, Falt $P$, Gallittu $P$, et al. Impact of carbon dioxide insufflation and water exchange on postcolonoscopy outcomes in patients receiving on-demand sedation: a randomized controlled trial. Gastrointest Endosc 2017; 85: 210-218.
8. Tang Z, Zhang DS, Thrift AP, et al. Impact of cap-assisted colonoscopy on the learning curve and quality in colonoscopy: a randomized controlled trial. Gastrointest Endosc 2018; 87: 723-732.

9. Kim SY, Chung JW, Park DK, et al. Comparison of carbon dioxide and air insufflation during consecutive EGD and colonoscopy in moderate-sedation patients: a prospective, double-blind, randomized controlled trial. Gastrointest Endosc 2017; 85: 12551262.

10. Sofi AA, Nawras A, Khan MA, et al. Meta-analysis of the performance of ultrathin vs. standard colonoscopes. Endoscopy 2017; 49: 351-358

11. Sato K, Ito $S$, Kitagawa $T$, et al. A prospective randomized study of the use of an ultrathin colonoscope versus a pediatric colonoscope in sedation-optional colonoscopy. Surg Endosc 2017; 31: 5150-5158.

12. Ogawa T, Ohda $Y$, Nagase $K$, et al. Evaluation of discomfort during colonoscopy with conventional and ultrathin colonoscopes in ulcerative colitis patients. Dig Endosc 2015; 27: 99-105.

13. Sato K, Shigiyama F, Ito $S$, et al. Colonoscopy using a smallcaliber colonoscope with passive-bending after incomplete colonoscopy due to sharp angulation or pain. Surg Endosc 2013; 27: 4171-4176.

14. Nemoto D, Isohata N, Utano K, et al. Double-balloon colonoscopy carried out by a trainee after incomplete conventional colonoscopy. Dig Endosc 2014; 26: 392-395.

15. Noda K, Kitada T, Suzuki Y, et al. A novel physical colonoscopy simulator based on analysis of data from computed tomography colonography. Surg Today 2017; 47: 1153-1162.

Journal of the Anus, Rectum and Colon is an Open Access journal distributed under the Creative Commons Attribution-NonCommercial-NoDerivatives 4.0 International License. To view the details of this license, please visit (https://creativ ecommons.org/licenses/by-nc-nd/4.0/). 